\title{
Electronic Computer Solution for the MPN Equation used in the Determination of Bacterial Populations
}

\author{
R. L. Norman and L. L. Kempe, The University of Michigan, Ann \\ Arbor, Michigan
}

Summary. The utility and the practical precision of the Most Probable Number method for determining bacterial populations is currently restricted by a lack of adequate tables of solved examples or alternatively by an easy and quick solution to individual problems. Where an electronic computer is available, these restrictions need not apply. A computer programme and example solution for tabulated data and individual problems are presented in this paper.

\section{Introduction}

The extinction dilution technique is one of two standard methods for the determination of the viable population of bacteria in a sample of material. ${ }^{3}$ As has been pointed out, ${ }^{1}$ this method developed from the Phelps Index, through McCrady's calculations, to the generalized relations of Halvorson and Ziegler. ${ }^{1}$ These latter, rather elegant equations, have been solved for only a few combinations of numbers, which in addition have been limited to decimal dilutions and small numbers of tubes in each dilution.

Halvorson and Ziegler ${ }^{2}$ pointed out that deviation from the mode is high using only five tubes in each of three decimal dilutions, but narrows rapidly with increased numbers of tubes in each dilution. Unfortunately, practical application of the Most Probable Number (MPN) method is severely restricted by the few tabulated solutions of the general equations that are available. Actually, only the tables for five tubes ${ }^{3}$ and for ten tubes in decimal dilutions are readily accessible, ${ }^{1}$ although solutions for a 
few other miscellaneous combinations can be obtained. ${ }^{3}$ Since this is only a restriction of equation solutions and not an inherent defect of the method, increased value of the MPN technique should result from the availability of other solutions. This paper presents an electronic computer programme capable of solving any reasonable combination of like numbers of tubes in decimal or other manner of dilutions.

\section{Theory}

Let several dilutions be used and several tubes be inoculated with each dilution of a bacterial culture. Halvorson and Ziegler's notations and equations are used, where:

$X=$ number of bacteria per $\mathrm{ml}$ in the median significant dilution,

$n_{1}=$ number of samples of volume $a_{1} \mathrm{ml}$ taken,

$p_{1}=$ number of failures out of $n_{1}$ trials, where failure means growth in the tubes,

$q_{1}=$ the number of successes out of $n_{1}$ trials, where success means no growth in the tubes.

If the set of tubes are inoculated with $a_{1} \mathrm{ml}$ in each of the $n_{1}$ tubes in set 1, $a_{2} \mathrm{ml}$ in the $n_{2}$ tubes of set 2 and so on; and if $p_{1}$ of the tubes of set 1 show growth, $p_{2}$ of set $2, \ldots$ where $a_{i}, n_{i}$ and $p_{i}$ refer to the $i$ th dilution, then a generalized form of Halvorson and Ziegler's equation gives :

$$
\sum_{i}^{N} a_{i}\left(\frac{p_{i}}{1-\mathrm{e}^{-a_{i} X}}-n_{i}\right)=0
$$

In addition, the percentage probability that this growth pattern will occur for the computed value of $X$ is obtained from:

$\log _{\mathrm{e}} P=\sum_{i}^{N}\left[\log _{\mathrm{e}}\left(\frac{n_{i} !}{p_{i} !\left(n_{i}-p_{i}\right) !}\right)-a_{i}\left(n_{i}-p_{i}\right) X+a_{i} p_{i} \log _{\mathrm{e}}\left(1-\mathrm{e}^{-a_{i} X}\right)\right]$

Since the statistical equation (1) is implicit in the desired unknown, $X$, it is most easily solved by an iterative, successive 
approximation method. In this case the Newton-Raphson method was used. Here a new approximation $\bar{X}$ to the unknown $X$ is generated with each iterative cycle from

$$
\bar{X}=X-\frac{G(X)}{\bar{d} \bar{G} / \bar{d} X}
$$

until $\bar{X}=X$ is established within a previously allowable error. Specifically, for this problem

and

$$
\begin{gathered}
G(X)=\sum_{i}^{N} a_{i}\left(\frac{p_{i}}{1-\mathrm{e}^{-a_{i} X}}-n_{i}\right) \\
\frac{d G}{d X}=-\sum_{i}^{N} \frac{a_{i}^{2} p_{i} \mathrm{e}^{-a_{i} X}}{\left(1-\mathrm{e}^{-a_{i} X}\right)^{2}}
\end{gathered}
$$

Using the above equations, the computer flow diagram shown in Fig. 1 was developed.

A programme to fit a computer was then produced from this flow sheet. The actual machine programme used depends upon the computer available; we used an *IBM-650 equipped with index accumulator and floating point accessories.

\section{Results and Discussion}

The programme described above can be used to solve quickly individual problems or to prepare tables of solutions. For example, a complete table containing all possible combinations of ten tubes in decimal dilutions was solved for $X$ and $P$ in $1 \mathrm{~h} 20 \mathrm{~min}$ of computer time. A condensed version of this table is given by Halvorson and Ziegler. ${ }^{1}$ The machine solutions were identical with those given in the above article except for one or two numbers. For example, at code $(10,2,0)$ the machine solution is $X=0.329$ and $P=12.53$ per cent, the published data show $X=0.329$ and $P=18 \cdot 14$. In this case, if the middle dilution, which is the median significant dilution, were $0.1 \mathrm{ml}$ then $x$, the number of bacteria per $\mathrm{ml}$ in the original undiluted sample, would be 0.329 multiplied by the reciprocal of the dilution or 3.29 bacteria per $\mathrm{ml}$.

\footnotetext{
* The detailed programme can be furnished upon request to the authors.
} 


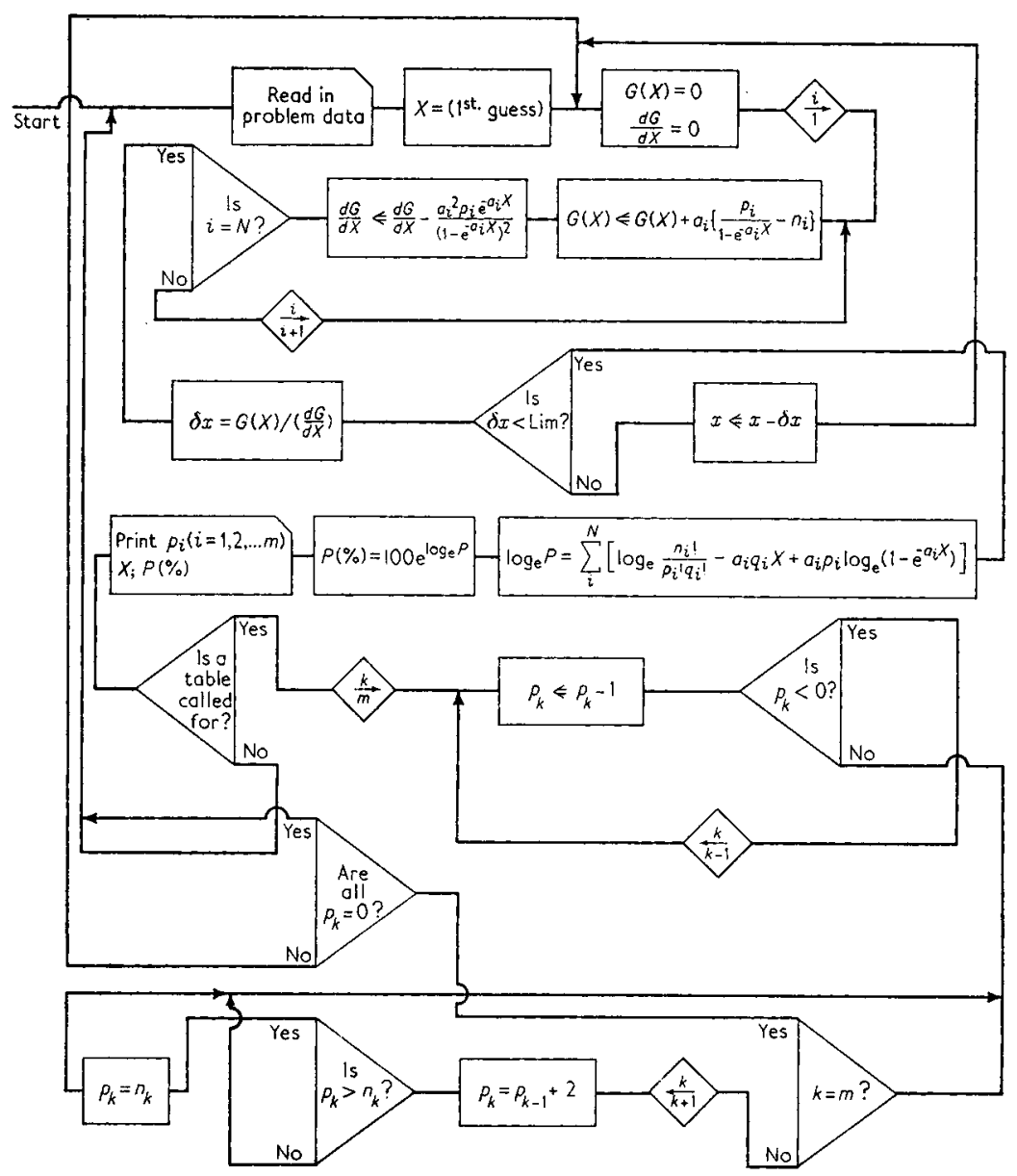

Giossary of Computer Symbols

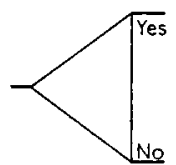

Switch in progromme bosed upon whether or not the condition described within the triangle is met or not met, os answered by yes or no

$\leftarrow$ means 'replaced by' i.e. the value of $X$ is replaced by $X-\delta X$. In this particular problem $\delta X$ is defined by equation 3 and numerically equal to $G(x)$ $\frac{G(X)}{(d G / d X)}$

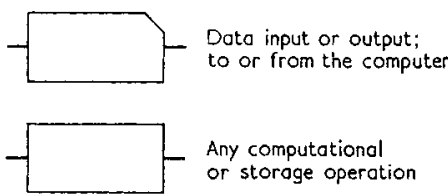

Repiacement, by the computer, of the index value named above the arrow with the one indicated below the arrow; for example $i$ with $i+1$, $k$ with $m$, etc.

Fig. 1. Computer programme for solution of equations for determination of the Most Probable Number of bacteria in a sample and of probabilities for occurrence of the indicated codes of bacterial growth in the culture tubes 
Results having a $P$ greater than 0.0049 for eight tubes in each of three decimal dilutions are given in Table I. As shown by the calculations of Halvorson and Ziegler, ${ }^{2}$ this selection of tubes and dilutions provides a considerable improvement in the anticipated precision for the calculated value of $X$ with a minimum of additional laboratory effort. Specifically, the deviations shown by Halvorson and Ziegler ${ }^{2}$ for 97 per cent of the data indicate an improvement for eight tubes as compared with five tubes per dilution as follows: with eight tubes the deviations above and below the mode are 155 and 63 per cent respectively, while with five tubes the values are 260 and 70 per cent. Obviously an even larger number of tubes improves the precision further; but at ten tubes the increase is small, the values being 130 per cent above and 58 per cent below the mode respectively. Hence, routine determinations of the most probable number of bacteria by the dilution method would materially benefit from the use of eight tubes in each of three dilutions rather than the customary five tubes. For this reason a table of solutions for the most probable number of bacteria present in the median significant dilution, using eight tubes in each of three decimal dilutions, is included in this paper.

Table I. The most probable numbers of bacteria per $\mathrm{ml}$ of the median dilution of sample using eight tubes in each of the decimal dilutions

\begin{tabular}{|c|c|c|c|c|c|c|c|c|c|}
\hline \multicolumn{3}{|c|}{ Code } & \multirow{2}{*}{$\frac{X}{20 \cdot 8}$} & \multirow{2}{*}{$\begin{array}{r}P \\
39 \cdot 3\end{array}$} & \multicolumn{3}{|c|}{ Code } & \multirow{2}{*}{$\frac{X}{2 \cdot 84}$} & \multirow{2}{*}{$\begin{array}{c}P \\
0.02\end{array}$} \\
\hline 8 & 8 & 7 & & & 8 & 6 & 6 & & \\
\hline 8 & 8 & 6 & 13.9 & $31 \cdot 1$ & 8 & 6 & 5 & $2 \cdot 50$ & $0 \cdot 16$ \\
\hline 8 & 8 & 5 & $9 \cdot 82$ & $28 \cdot 2$ & 8 & 6 & 4 & $2 \cdot 18$ & 0.75 \\
\hline 8 & 8 & 4 & $7 \cdot 02$ & $27 \cdot 1$ & 8 & 6 & 3 & $1 \cdot 89$ & $2 \cdot 66$ \\
\hline 8 & 8 & 3 & $5 \cdot 10$ & $26 \cdot 5$ & 8 & 6 & 2 & $1 \cdot 63$ & 6.9 .2 \\
\hline 8 & 8 & 2 & $3 \cdot 85$ & $23 \cdot 9$ & 8 & 6 & 1 & $1 \cdot 38$ & $12 \cdot 22$ \\
\hline 8 & 8 & 1 & $3 \cdot 01$ & $16 \cdot 9$ & 8 & 6 & 0 & $1 \cdot 15$ & $11 \cdot 39$ \\
\hline 8 & 8 & 0 & $2 \cdot 40$ & 6.85 & 8 & 5 & 6 & $2 \cdot 13$ & 0.00 \\
\hline 8 & 7 & 8 & 5.96 & 0.00 & 8 & 5 & 5 & $1 \cdot 89$ & 0.04 \\
\hline 8 & 7 & 7 & $5 \cdot 08$ & 0.04 & 8 & 5 & 4 & $1 \cdot 66$ & 0.26 \\
\hline 8 & 7 & 6 & $4 \cdot 33$ & 0.21 & 8 & 5 & 3 & $1 \cdot 44$ & $1 \cdot 27$ \\
\hline 8 & 7 & 5 & $3 \cdot 69$ & 0.87 & 8 & 5 & 2 & $1 \cdot 23$ & $4 \cdot 45$ \\
\hline 8 & 7 & 4 & $3 \cdot 14$ & $2 \cdot 67$ & 8 & 5 & 1 & 1.030 & $10 \cdot 66$ \\
\hline 8 & 7 & 3 & $2 \cdot 67$ & $6 \cdot 36$ & 8 & 5 & 0 & 0.842 & $13 \cdot 61$ \\
\hline 8 & 7 & 2 & $2 \cdot 26$ & 11.40 & 8 & 4 & 5 & 1.48 & 0.01 \\
\hline 8 & 7 & 1 & 1.91 & $14 \cdot 10$ & 8 & 4 & 4 & $1 \cdot 30$ & $0 \cdot 10$ \\
\hline 8 & 7 & 0 & 1.59 & $9 \cdot 26$ & 8 & 4 & 3 & $1 \cdot 11$ & 0.62 \\
\hline
\end{tabular}


Table I. Continued

\begin{tabular}{|c|c|c|c|c|c|c|c|c|c|}
\hline \multicolumn{3}{|c|}{ Codo } & \multirow{2}{*}{$\frac{X}{0.940}$} & \multirow{2}{*}{$\frac{P}{2 \cdot 88}$} & \multicolumn{3}{|c|}{ Code } & \multirow{2}{*}{$\frac{X}{0 \cdot 155}$} & \multirow{2}{*}{$\frac{P}{8 \cdot 17}$} \\
\hline 8 & 4 & 2 & & & 7 & 0 & 0 & & \\
\hline 8 & 4 & 1 & 0.774 & $9 \cdot 22$ & 6 & 6 & 1 & 0.308 & $0 \cdot 00$ \\
\hline 8 & 4 & 0 & 0.622 & $16 \cdot 01$ & 6 & 6 & 0 & 0.277 & 0.02 \\
\hline 8 & 3 & 5 & $1 \cdot 18$ & 0.00 & 6 & 5 & 1 & 0.273 & 0.03 \\
\hline 8 & $\mathbf{3}$ & 4 & 1.02 & 0.04 & 6 & 5 & 0 & 0.244 & $0 \cdot 13$ \\
\hline 8 & 3 & 3 & 0.867 & $0 \cdot 30$ & 6 & 4 & 2 & 0.269 & 0.01 \\
\hline 8 & 3 & 2 & 0.718 & $1 \cdot 80$ & 6 & 4 & 1 & 0.241 & $0 \cdot 12$ \\
\hline 8 & 3 & 1 & 0.582 & $7 \cdot 70$ & 6 & 4 & 0 & 0.214 & 0.63 \\
\hline 8 & 3 & 0 & 0.467 & 17.98 & 6 & 3 & 2 & 0.238 & 0.03 \\
\hline 8 & 2 & 4 & 0.807 & 0.01 & 6 & 3 & 1 & 0.211 & 0.37 \\
\hline 8 & 2 & 3 & 0.672 & 0.13 & 6 & 3 & 0 & $0 \cdot 186$ & $2 \cdot 29$ \\
\hline 8 & 2 & 2 & 0.550 & $1 \cdot 03$ & 6 & 2 & 2 & 0.209 & 0.06 \\
\hline 8 & 2 & 1 & 0.445 & 5.81 & 6 & 2 & 1 & $0 \cdot 184$ & 0.84 \\
\hline 8 & 2 & 0 & 0.362 & $17 \cdot 73$ & 6 & 2 & 0 & 0.160 & 6.08 \\
\hline 8 & 1 & 3 & 0.522 & 0.05 & 6 & 1 & 2 & 0.182 & 0.08 \\
\hline 8 & 1 & 2 & 0.427 & 0.47 & 6 & 1 & 1 & $0 \cdot 158$ & $1 \cdot 29$ \\
\hline 8 & 1 & 1 & 0.350 & $3 \cdot 44$ & 6 & 1 & 0 & $0 \cdot 135$ & $10 \cdot 95$ \\
\hline 8 & 1 & 0 & 0.287 & $13 \cdot 34$ & 6 & 0 & 2 & 0.156 & 0.05 \\
\hline 8 & 0 & 2 & 0.338 & $0 \cdot 13$ & 6 & 0 & 1 & $0 \cdot 134$ & $1 \cdot 03$ \\
\hline 8 & 0 & 1 & 0.280 & $1 \cdot 17$ & 6 & 0 & 0 & 0.113 & $10 \cdot 40$ \\
\hline 8 & 0 & 0 & 0.231 & $5 \cdot 67$ & 5 & 5 & 1 & 0.207 & 0.01 \\
\hline 7 & 7 & 1 & 0.547 & 0.01 & 5 & $\mathbf{5}$ & 0 & $0 \cdot 185$ & 0.04 \\
\hline 7 & 7 & 0 & 0.484 & 0.02 & 5 & 4 & 1 & $0 \cdot 184$ & 0.03 \\
\hline 7 & 6 & 2 & 0.530 & $0 \cdot 01$ & 5 & 4 & 0 & $0 \cdot 163$ & 0.23 \\
\hline 7 & 6 & 1 & 0.471 & 0.05 & 5 & 3 & 2 & $0 \cdot 182$ & 0.01 \\
\hline 7 & 6 & 0 & 0.415 & 0.15 & 5 & 3 & 1 & $0 \cdot 161$ & $0 \cdot 14$ \\
\hline 7 & 5 & 2 & 0.458 & 0.03 & 5 & 3 & 0 & $0 \cdot 141$ & $1 \cdot 13$ \\
\hline 7 & 5 & 1 & 0.404 & $0 \cdot 20$ & 5 & 2 & 2 & 0.160 & 0.02 \\
\hline 7 & 5 & 0 & $0 \cdot 355$ & 0.64 & 5 & 2 & I & $0 \cdot 140$ & 0.42 \\
\hline 7 & 4 & 3 & $0 \cdot 446$ & 0.01 & 5 & 2 & 0 & 0.121 & $4 \cdot 04$ \\
\hline 7 & 4 & 2 & 0.395 & 0.07 & 5 & 1 & 2 & $0 \cdot 139$ & 0.04 \\
\hline 7 & 4 & 1 & $0 \cdot 347$ & 0.55 & 5 & 1 & 1 & $0 \cdot 120$ & 0.87 \\
\hline 7 & 4 & 0 & 0.304 & 2.09 & 5 & 1 & 0 & 0.101 & $9 \cdot 83$ \\
\hline 7 & 3 & 3 & 0.386 & 0.01 & 5 & 0 & 2 & 0.119 & 0.04 \\
\hline 7 & 3 & 2 & $0 \cdot 340$ & 0.13 & 5 & 0 & 1 & $0 \cdot 101$ & 0.94 \\
\hline 7 & 3 & 1 & 0.298 & $1 \cdot 16$ & 5 & 0 & 0 & 0.083 & $12 \cdot 75$ \\
\hline 7 & 3 & 0 & 0.259 & $5 \cdot 15$ & 4 & 4 & 0 & $0 \cdot 128$ & $0 \cdot 09$ \\
\hline 7 & 2 & 3 & $0 \cdot 333$ & 0.01 & 4 & 3 & 1 & $0 \cdot 127$ & 0.05 \\
\hline 7 & 2 & 2 & 0.292 & 0.18 & 4 & 3 & 0 & 0.110 & 0.57 \\
\hline 7 & 2 & 1 & 0.255 & $1 \cdot 83$ & 4 & 2 & 1 & $0 \cdot 109$ & 0.22 \\
\hline 7 & 2 & 0 & 0.220 & 9.53 & 4 & 2 & 0 & 0.093 & $2 \cdot 67$ \\
\hline 7 & 1 & 3 & 0.287 & 0.01 & 4 & 1 & 2 & $0 \cdot 108$ & 0.02 \\
\hline 7 & l & 2 & 0.251 & $0 \cdot 16$ & 4 & 1 & l & 0.092 & 0.59 \\
\hline 7 & l & $\mathbf{l}$ & 0.217 & 1.97 & 4 & 1 & 0 & 0.076 & $8 \cdot 70$ \\
\hline 7 & 1 & 0 & 0.186 & $12 \cdot 12$ & 4 & 0 & 2 & 0.091 & 0.02 \\
\hline 7 & 0 & 2 & 0.214 & 0.08 & 4 & 0 & 1 & 0.075 & 0.84 \\
\hline 7 & 0 & 1 & 0.183 & $1 \cdot 11$ & 4 & 0 & 0 & 0.060 & 15.52 \\
\hline
\end{tabular}


Table I. Continued

\begin{tabular}{|c|c|c|c|c|c|c|c|c|c|}
\hline \multicolumn{3}{|c|}{ Code } & \multirow{2}{*}{$\frac{X}{0 \cdot 101}$} & \multirow{2}{*}{$\frac{P}{0.03}$} & \multicolumn{3}{|c|}{ Code } & \multirow{2}{*}{$\frac{X}{0 \cdot 039}$} & \multirow{2}{*}{$\begin{array}{c}P \\
6.35\end{array}$} \\
\hline $\mathbf{3}$ & 4 & 0 & & & 2 & 1 & 0 & & \\
\hline 3 & 3 & 1 & $0 \cdot 100$ & 0.02 & 2 & 0 & 2 & 0.051 & 0.01 \\
\hline 3 & 3 & 0 & 0.085 & 0.28 & 2 & 0 & 1 & 0.038 & $0 \cdot 62$ \\
\hline 3 & 2 & 1 & 0.085 & 0.11 & 2 & 0 & 0 & 0.026 & $24 \cdot 54$ \\
\hline 3 & 2 & 0 & 0.070 & $1 \cdot 72$ & 1 & 3 & 0 & 0.049 & 0.05 \\
\hline 3 & 1 & 2 & 0.084 & 0.01 & 1 & 2 & 1 & 0.049 & 0.02 \\
\hline 3 & 1 & 1 & 0.070 & $0 \cdot 38$ & 1 & 2 & 0 & 0.036 & 0.53 \\
\hline 3 & 1 & 0 & 0.056 & $7 \cdot 56$ & 1 & 1 & 1 & 0.036 & 0.12 \\
\hline 3 & 0 & 2 & 0.069 & 0.02 & 1 & 1 & 0 & 0.024 & $5 \cdot 00$ \\
\hline 3 & 0 & 1 & 0.055 & 0.74 & 1 & 0 & 2 & 0.036 & 0.01 \\
\hline 3 & 0 & 0 & 0.041 & $19 \cdot 12$ & 1 & 0 & 1 & 0.024 & 0.50 \\
\hline 2 & 4 & 0 & 0.079 & 0.01 & 1 & 0 & 0 & 0.012 & $35 \cdot 14$ \\
\hline 2 & 3 & 1 & 0.079 & 0.01 & 0 & 2 & 0 & 0.023 & 0.19 \\
\hline 2 & 3 & 0 & 0.066 & $0 \cdot 12$ & 0 & 1 & 1 & 0.023 & 0.04 \\
\hline 2 & 2 & 1 & 0.065 & $0 \cdot 05$ & 0 & 1 & 0 & 0.011 & $\mathbf{3} \cdot \mathbf{3 3}$ \\
\hline 2 & 2 & 0 & $0 \cdot 052$ & $1 \cdot 03$ & 0 & 0 & 1 & 0.011 & 0.33 \\
\hline 2 & 1 & 1 & 0.052 & $0 \cdot 23$ & & & & & \\
\hline
\end{tabular}

To use this table for determining bacterial populations, Halvorson's ${ }^{1}$ notations apply. In each dilution, the code refers to the number of tubes showing growth out of eight tubes inoculated. For example, if eight tubes of suitable liquid media are inoculated with $1 \mathrm{ml}$, eight with $0.1 \mathrm{ml}$ and eight with $0.01 \mathrm{ml}$ of sample and growth occurs in seven, five, and one tube out of each set upon incubation, then 0.404 bacteria were present per $\mathrm{ml}$ of the median dilution or 4.04 bacteria per $\mathrm{ml}$ of undiluted sample. The probability of this combination of tubes developing is 0.20 per cent from an infinite number of trials using a suspension containing 4.04 bacteria per $\mathrm{ml}$. It should be noted that $X=x$ when the median significant dilution is 1 , i.e. not diluted; for other median significant dilutions, $x$ equals $X$ divided by the fractional value of this dilution. In the above instance $(0 \cdot 404)$ $(1 / 0 \cdot 1)=4 \cdot 04$ bacteria per $\mathrm{ml}$ of the original sample

The equations, and therefore the machine solutions, are not restricted to decimal dilutions or to any prescribed number of tubes per dilution. For example, a table could be developed using 15 tubes per dilution, five such dilutions and a two-fold ratio between sets of tubes; or if enough similar problems were available requiring solution to warrant the use of a computer, they could be readily and quickly solved.

\section{References}

1 Halvorson, H. O. and Ziegler, N. R. J. Bact., 25, 101-121 (1933)

2 Halvorson, H. O. and Ziegler, N. R. J. Bact., 26, 559-567 (1933)

3 Standard Methods for the Examination of Water, Sewage and Industrial Wastes, 10th ed., American Public Health Association 